\title{
Author Correction: A practical guide for mutational signature analysis in hematological malignancies
}

\author{
Francesco Maura 1,2,3, Andrea Degasperi (3) 3,4, Ferran Nadeu (10 6,7, Daniel Leongamornlert ${ }^{3}$, Helen Davies 3,4,5, \\ Luiza Moore (10 ${ }^{3}$, Romina Royo ${ }^{8}$, Bachisio Ziccheddu ${ }^{9}$, Xose S. Puente (10) ${ }^{10,11}$, Herve Avet-Loiseau ${ }^{12}$, \\ Peter J. Campbell ${ }^{3}$, Serena Nik-Zainal ${ }^{3,4,5}$, Elias Campo ${ }^{6,7,8}$, Nikhil Munshi ${ }^{13,14}$ \& Niccolò Bolli ${ }^{2,9}$
}

Correction to: Nature Communications https://doi.org/10.1038/s41467-019-11037-8, published online 5 July 2019.

The original version of this Article contained an error in the spelling of the author Peter J Campbell, which was incorrectly given as Peter J Cambell. This has now been corrected in both the PDF and HTML versions of the Article.

Published online: 25 July 2019

\begin{abstract}
(c) (i) Open Access This article is licensed under a Creative Commons Attribution 4.0 International License, which permits use, sharing, adaptation, distribution and reproduction in any medium or format, as long as you give appropriate credit to the original author(s) and the source, provide a link to the Creative Commons license, and indicate if changes were made. The images or other third party material in this article are included in the article's Creative Commons license, unless indicated otherwise in a credit line to the material. If material is not included in the article's Creative Commons license and your intended use is not permitted by statutory regulation or exceeds the permitted use, you will need to obtain permission directly from the copyright holder. To view a copy of this license, visit http://creativecommons.org/licenses/by/4.0/.
\end{abstract}

(C) The Author(s) 2019

\footnotetext{
${ }^{1}$ Myeloma Service, Department of Medicine, Memorial Sloan Kettering Cancer Center, New York 10065 NY, USA. ${ }^{2}$ Department of Oncology and HematoOncology, University of Milan, Via Festa del Perdono 7, Milan 20122, Italy. ${ }^{3}$ Cancer, Ageing, and Somatic Mutation Programme, Wellcome Sanger Institute, Hinxton, Cambridgeshire CB10 1SA, UK. ${ }^{4}$ Department of Medical Genetics, Cambridge University Hospitals NHS Foundation Trust, Cambridge CB2 0QQ, UK. ${ }^{5}$ MRC Cancer Unit, University of Cambridge, Hutchison/MRC Research Centre, Cambridge Biomedical Campus, Cambridge CB2 OXZ, UK. ${ }^{6}$ Patologia Molecular de Neoplàsies Limfoides, Institut d'Investigacions Biomèdiques August Pi i Sunyer (IDIBAPS), 08036 Barcelona, Spain. ${ }^{7}$ Centro de Investigación Biomédica en Red de Cáncer (CIBERONC), 28029 Madrid, Spain. ${ }^{8}$ Barcelona Supercomputing Center (BSC), Joint BSC-CRG-IRB Research Program in Computational Biology, 08036 Barcelona, Spain. ${ }^{9}$ Department of Clinical Oncology and Hematology, Fondazione IRCCS Istituto Nazionale dei Tumori, Milan 20133, Italy. ${ }^{10}$ Unitat Hematopatologia, Hospital Clínic of Barcelona, Universitat de Barcelona, 08036 Barcelona, Spain. ${ }^{11}$ Departamento de Bioquimica y Biologia Molecular, Instituto Universitario de Oncologia (IUOPA), Universidad de Oviedo, Oviedo 33003, Spain. ${ }^{12}$ IUC-Oncopole, and CRCT INSERM U1037, 31100 Toulouse, France. ${ }^{13}$ Jerome Lipper Multiple Myeloma Center, Dana-Farber Cancer Institute, Harvard Medical School, Boston 02215 MA, USA.

${ }^{14}$ Veterans Administration Boston Healthcare System, West Roxbury 02130 MA, USA. Correspondence and requests for materials should be addressed to F.M. (email: mauraf@mskcc.org) or to N.B. (email: niccolo.bolli@unimi.it)
} 